\title{
NOTES
}

\section{Comparison of Free-Volume Theories}

\author{
J. S. VRentas, C. M. VRentas, and J. L. Duda \\ Department of Chemical Enigineering, The Pennsylvania State University, \\ University Park, PA 16802, U.S.A.
}

(Received June 10, 1992)

\begin{abstract}
KEY WORDS Free-Volume Theories / Fujita Theory / Vrentas-Duda Theory / Jump Units / Sufficient Conditions / Self-Diffusion Coefficients / Fractional Hole Free Volume / Critical Hole Volume /
\end{abstract}

In a recent paper, ${ }^{1}$ Fujita has presented a useful comparison of the free-volume theories of Fujita ${ }^{2}$ and of Vrentas and Duda. ${ }^{3-6}$ Fujita has stated that an important difference in the theories is that the Fujita theory is based on the utilization of the free volume per unit volume of solution whereas the VrentasDuda theory is based on the utilization of the average free volume per jumping unit. This statement is of course correct, as can be easily seen by consulting the original papers. However, Fujita also questioned the VrentasDuda conclusion ${ }^{5}$ that the Fujita theory can be regarded as a restricted form of the Vrentas-Duda theory. The purpose of this note is to show that this original conclusion is indeed correct.

\section{THEORY}

From the Vrentas-Duda theory, ${ }^{3-6}$ the following equation can be derived ${ }^{7}$

$$
\begin{gathered}
\ln \left[\frac{D_{1}}{D_{1}(0)}\right]=\frac{\phi_{1} F}{\left[\frac{f_{2}^{2}}{\gamma^{2}}+\phi_{1} \frac{f_{2}}{\gamma}\left(\frac{f_{1}}{\gamma}-\frac{f_{2}}{\gamma}\right)\right]} \\
F=\frac{\xi \hat{V}_{2}^{*} f_{1}}{\hat{V}_{2}^{0} \gamma}-\frac{\hat{V}_{1}^{*} f_{2}}{\hat{V}_{1}^{0} \gamma}
\end{gathered}
$$

where $D_{1}$ is the solvent self-diffusion coefficient, $D_{1}(0)$ refers to the value of $D_{1}$ evaluated at zero solvent concentration, $\phi_{I}$ is the volume fraction of component $I$, and $f_{I}$ is the fractional hole free volume of pure component $I$ at the temperature of interest. Also, $\hat{V}_{I}^{*}$ is the specific critical hole free volume of component $I$ required for a jump, $\xi$ is the ratio of the critical molar volume of the solvent jumping unit to the critical molar volume of the polymer jumping unit, $\hat{V}_{I}^{0}$ is the specific volume of pure component $I$ at the temperature of interest, and $\gamma$ is an overlap factor for free volume. The above equation is based on the assumption that the partial specific volumes of the solvent and polymer are independent of composition so that there is no volume change on mixing. Furthermore, for the Fujita theory, it is easy to derive the following result ${ }^{7}$

$$
\ln \left[\frac{D_{1}}{D_{1}(0)}\right]=\frac{B_{\mathrm{d}}\left(\bar{f}_{1}-\bar{f}_{2}\right) \phi_{1}}{\left[\bar{f}_{2}^{2}+\bar{f}_{2} \phi_{1}\left(\bar{f}_{1}-\bar{f}_{2}\right)\right]}
$$

where the free-volume parameters of the theory are $B_{\mathrm{d}}, \bar{f}_{1}$, and $\bar{f}_{2}$. In the correlative version of the Fujita theory, $\bar{f}_{1} / B_{\mathrm{d}}$ and $\bar{f}_{2} / \boldsymbol{B}_{\mathrm{d}}$ can be determined from diffusivity data, and it is not necessary to give a physical interpretation to these parameters. However, to carry out a comparison of eq 1 and 3 , it is convenient to introduce the following equalities:

$$
\begin{aligned}
& f_{1}=\bar{f}_{1} \\
& f_{2}=\bar{f}_{2}
\end{aligned}
$$


It is clear from a comparison of eq 1 and 3 that the Fujita and Vrentas-Duda theories are identical when the following sufficient condition is satisfied

$$
\frac{\xi \hat{V}_{2}^{*}}{\hat{V}_{2}^{0}}=\frac{\hat{V}_{1}^{*}}{\hat{V}_{1}^{0}}
$$

and when $B_{\mathrm{d}}$ is defined as follows:

$$
B_{\mathrm{d}}=\frac{\gamma \hat{V}_{1}^{*}}{\hat{V}_{1}^{0}}
$$

In a previous study, ${ }^{5}$ it was stated that sufficient conditions for showing that the Vrentas-Duda theory reduces to the Fujita theory are the following

$$
\begin{gathered}
\frac{\omega_{1}\left(\hat{V}_{1}^{0}-\hat{V}_{2}^{0}\right)}{\hat{V}_{2}^{0}}<<1 \\
\xi \hat{V}_{2}^{*}=\hat{V}_{1}^{*}
\end{gathered}
$$

where $\omega_{1}$ is the mass fraction of solvent. Clearly, eq 8 will be valid over the complete concentration range if

$$
\hat{V}_{1}^{0}=\hat{V}_{2}^{0}
$$

It is evident that eq 6 is a sufficient condition which represents a compact version of the two previous sufficient conditions, eq 9 and 10 . It should be clear then that the Vrentas-Duda theory reduces to the Fujita theory when certain restrictions are imposed. Consequently, the Fujita theory can be regarded, in a sense, as a restricted form of the Vrentas-Duda theory, and we hence believe that our previous conclusion $^{5}$ is correct.

If the Vrentas-Duda extension of the freevolume theory from pure materials to binary mixtures is correct, then the Fujita theory is a special result which will be valid only for a restricted set of conditions. On the other hand, if the Fujita extension of the freevolume theory to solutions is appropriate, then the Vrentas-Duda theory will produce correct results only for a restricted set of conditions. We believe that our extension of free-volume theory to binary solutions pro- vides a correct generalization of the freevolume theory of transport, and there is some evidence which supports this conclusion. ${ }^{3}$ It seems to us that an extension of the pure component free-volume theory to binary solutions must be based on the determination of the free volume associated with each jumping unit. Consequently, we have in previous work taken the position that the Vrentas-Duda theory is the general result, and the Fujita theory is simply a special case of this more general theory.

To summarize, the above results show that the Fujita theory can be derived when certain restrictions are imposed on the Vrentas-Duda theory, and hence we believe that our original conclusion $^{5}$ on this point is correct. Certainly, the original assumptions of the Fujita and Vrentas-Duda theories are different, as Fujita has stated, but the equations of the two theories can be made identical by imposing certain restrictions on the Vrentas-Duda equation. Finally, this can also be seen quite nicely by considering the equations for $D_{1}$ without using the diffusivity ratio form. For the Vrentas-Duda theory, $D_{1}$ is given by the equation

$$
\ln D_{1}=\ln D_{0}-\frac{E}{R T}-\frac{\gamma\left(\phi_{1} \frac{\hat{V}_{1}^{*}}{\hat{V}_{1}^{0}}+\phi_{2} \frac{\hat{V}_{2}^{*} \xi}{\hat{V}_{2}^{0}}\right)}{\phi_{1} f_{1}+\phi_{2} f_{2}}
$$

and, for the Fujita theory, we have the following expression:

$$
\ln D_{1}=\ln D_{0}-\frac{E}{R T}-\frac{B_{\mathrm{d}}}{\phi_{1} \bar{f}_{1}+\phi_{2} \bar{f}_{2}}
$$

The energy term has been added to the Fujita theory to facilitate comparison with the Vrentas-Duda theory. Here, $D_{0}$ is a constant preexponential factor, $E$ is the energy per mole needed to overcome attractive forces, and $T$ is temperature. Again, if eq 4 and 5 are used to facilitate comparison, then it is clear from eq 11 and 12 that the two theories give 
identical results when eq 6 is satisfied and when $B_{\mathrm{d}}$ is defined using eq 7 .

Finally, it is evident from the above discussion that the Vrentas-Duda theory permits any value of $\xi$ whereas the Fujita theory is restricted to a value of $\xi$ defined by eq 6. Since the Fujita theory follows from the Vrentas-Duda theory for a particular value of $\xi$, it seems reasonable to consider the Fujita theory as a special case of a theory which allows any value of $\xi$. Indeed, when eq 6 is satisfied, differences between a formulation based on the free volume per unit volume and a formulation based on the free volume per jumping unit effectively disappear. The question of whether the diffusion process can be described by a particular value of $\xi$ or whether a general value of $\xi$ is needed is best answered by a comparison of theory with experiment. Comparisons of the two theories with experiment $\mathrm{t}^{7-10}$ indicate that the Vrentas-Duda theory generally has superior predictive capabilities.
Acknowledgment. This work was supported by funds provided by the Dow Chemical Company.

\section{REFERENCES}

1. H. Fujita, Polym. J., 23, 1499 (1991).

2. H. Fujita, Fortsch. Hochpolym. Forsch., 3, 1 (1961).

3. J. S. Vrentas, J. L. Duda, and H.-C. Ling, J. Polym. Sci., Polym. Phys. Ed., 23, 275 (1985).

4. J. S. Vrentas, J. L. Duda, H.-C. Ling, and A.-C. Hou, J. Polym. Sci., Polym. Phys. Ed., 23, 289 (1985).

5. J. S. Vrentas and J. L. Duda, J. Polym. Sci., Polym. Phys. Ed., 15, 403 (1977).

6. J. S. Vrentas and J. L. Duda, J. Polym. Sci., Polym. Phys. Ed., 15, 417 (1977).

7. J. S. Vrentas and C. M. Vrentas, J. Polym. Sci., Polym. Phys. Ed., accepted for publication.

8. J. S. Vrentas and J. L. Duda, Macromolecules, 9, 785 (1976).

9. J. L. Duda, J. S. Vrentas, S. T. Ju, and H. T. Liu, AIChE J., 28, 279 (1982).

10. H. T. Liu, J. L. Duda, and J. S. Vrentas, Macromolecules, 13, 1587 (1980). 